\title{
Should Antibiotics Be Given to Children With Otitis Media to Prevent Mastoiditis?
}

Thompson PL, Gilbert RE, Long PF, et al.: Effect of antibiotics for otitis media on mastoiditis in children: a retrospective cohort study using the United Kingdom General Practice Research Database. Pediatrics 2009, 123:424-430.

Rating: $\bullet$ Of major importance.

Introduction: In the past, the prevention of the development of complications, including mastoiditis, was used as a rationale for the routine treatment of otitis media with antibiotics in children. This study sheds light on the appropriateness of that recommendation.

Aims: To determine if cases of mastoiditis have increased in association with the decline in antibiotics prescribed to children by primary care physicians in the United Kingdom.

Methods: Time trends in mastoiditis incidence, the frequency of antecedent otitis media, and the effect of antibiotics for otitis media on the risk of mastoiditis in children were examined. A retrospective cohort study was conducted using the United Kingdom General Practice Research Database, which included children 3 months to 15 years of age between 1990 and 2006. Risk of mastoiditis within 3 months after otitis media diagnosis and the protective effect of antibiotics were determined.

Results: Of the 2,622,348 children in the database, 854 had mastoiditis, only $35.7 \%$ of whom had antecedent otitis media. The incidence of mastoiditis remained stable during the study years at about 1.2 per 10,000 childyears. Overall, the risk of mastoiditis after otitis media was 1.8 per 10,000 episodes after antibiotics, compared with 3.8 per 10,000 without antibiotics. Although antibiotics halved the risk of mastoiditis, practitioners would need to treat 4831 otitis media episodes with antibiotics to prevent one child from developing mastoiditis. Viewed another way, if antibiotics were no longer prescribed for otitis media, an extra 255 cases of childhood mastoiditis would occur, but there would be 738,775 fewer antibiotic prescriptions per year in the United Kingdom.
Discussion: In the United Kingdom, most children with mastoiditis did not see their general practitioner for otitis media. Antibiotic use did halve the risk of mastoiditis, but the number of otitis media episodes needing treatment to prevent one case of mastoiditis was very high. Mastoiditis can be a serious disease, but most children make an uncomplicated recovery after mastoidectomy or use of intravenous antibiotics.

\section{Comments}

In contrast to views of the past, prevention of the development of mastoiditis does not appear to suffice as a rationale for the routine treatment of otitis media with antibiotics. Such routine treatment could pose a larger public health problem in terms of antibiotic resistance. The study's main strengths are the large size of the database and its national representativeness. Potential weaknesses include the case review and retrospective cohort study design.

\section{Acknowledgment}

Dr. David P. Skoner is affiliated with the Drexel University College of Medicine, where he is a Professor of Pediatrics; the West Virginia University School of Medicine, where he is a Clinical Professor of Pediatrics; and Allegheny General Hospital, where he is the Director of Allergy, Asthma, and Immunology. Correspondence should be sent to 320 East North Avenue, Pittsburgh, PA 15212, USA.E-mail: dskoner@wpahs.org.

\section{Disclosure}

Dr. Skoner has served on the speakers' bureau for AstraZeneca, Sanofi-Aventis, Merck \& Co., Schering-Plough, and Novartis Pharmaceuticals; has received grant research support from AstraZeneca, Sanofi-Aventis, Novartis Pharmaceuticals, and Schering-Plough; and has served as a consultant for Merck \& Co. 